\title{
Modeling of 3-stage Electromagnetic Induction Launcher
}

\author{
Daehwan Kwak ${ }^{1}$, Young Bae Kim², Jong Soo Kim², Chuhyun $\mathrm{Cho}^{2}$, Kyung-Seung Yang ${ }^{3}$, \\ Seong-Ho Kim ${ }^{3}$, Byung-Ha Lee ${ }^{3}$, Sanghyuk $\mathrm{An}^{3}$, Young-Hyun Lee ${ }^{3}$, Seok Han Yoon ${ }^{4}$, \\ In Su Koo ${ }^{4}$, Yong Gi Baik ${ }^{4}$, and Yun Sik Jin ${ }^{2 *}$ \\ ${ }^{1}$ Korea University of Science and Technology, 217 Gajeong-ro, Yuseong-gu, Daejeon, Korea \\ ${ }^{2}$ Korea Electrotechnology Research Institute, 12 Bulmosan-ro 10 beon-gil, Seongsan-gu, Changwon, Korea \\ ${ }^{3}$ Agency for Defense Development, P.O. Box 35, Yuseong-gu, Daejeon, Korea \\ ${ }^{4}$ Hyundaiwia Corporation, 153 Jungdong-ro, Seongsan-gu, Changwon, Korea
}

(Received 12 May 2015, Received in final form 4 September 2015, Accepted 9 September 2015)

\begin{abstract}
Electromagnetic Induction Launchers (EIL) have been receiving great attention due to their advantages of noncontact between the coils and a projectile. This paper describes the modeling and design of 3-stage EIL to accelerate a copper projectile of $50 \mathrm{~kg}$ with $290 \mathrm{~mm}$ diameter. Our EIL consists of three independent driving coils and pulsed power modules to generate separate driving currents. To find efficient acceleration conditions, the appropriate shape of the driving coils and the position of the projectile have been calculated by using a finite element analysis (FEA) method. The results showed that the projectile can be accelerated more effectively as the gap between the coils is smaller; a final velocity of $45 \mathrm{~m} / \mathrm{s}$ was obtained. The acceleration efficiency was estimated to be $23.4 \%$ when a total electrical energy of $216 \mathrm{~kJ}$ was discharged.
\end{abstract}

Keywords : induction launcher, mutual inductance, pulsed power, driving coils, finite element analysis

\section{Introduction}

The multi-stage electromagnetic induction launcher (EIL) accelerates a projectile using sequential repulsive forces between driving coils and induced current in the projectile [1]. The system is almost free from physical contact between the projectile and the barrel, so it has a longer launcher lifetime compared with other electromagnetic launchers. The potential applications of EIL include EM mortars [2], missile launchers [3], and countermeasure decoy launchers [4].

In order to design a multi-stage EIL system for a given projectile diameter and mass, parameters such as driving coil shape (number of turns, layers, coil diameters), starting position of the projectile, distance between coils, and trigger time of the pulsed power system should be optimized. Usually an analytic solution to describe the motion of the projectile in a multi-stage EIL is quite difficult to obtain. Accordingly, recent studies use FEA (finite element analysis) to design multi-stage EILs $[5,6]$. Usually, the FEAs were

(C)The Korean Magnetics Society. All rights reserved.

*Corresponding author: Tel: +82-55-280-1458

Fax: +82-55-280-1490, e-mail: ysjin@keri.re.kr performed with commercial software.

In this paper, we designed a 3-stage EIL by using an electromagnetic simulation program (Maxwell 2D, Ansys Electromagnetics Suite 16.2). The goal of our EIL is to accelerate a $50 \mathrm{~kg}$ with $290 \mathrm{~mm}$ in diameter copper projectile to a velocity greater than $30 \mathrm{~m} / \mathrm{s}$. We carried out detailed design of 3 driving coils and calculation of optimum trigger time. Notably, the simulation conditions of the driving coils were compressed through the calculation of $\mathrm{dM} / \mathrm{dz}$, axial gradient of mutual inductance. The simulation of 3 separate coils with an initial velocity condition was conducted first, and combined coil calculations with optimum trigger position against the moving projectile were performed.

\section{Design and Modelling}

2.1. Modeling of EIL structure and electrical circuits

The $50 \mathrm{~kg}$ disc shaped copper projectile had a diameter of $290 \mathrm{~mm}$ and a thickness of $90 \mathrm{~mm}$. The projectile is guided by a tube which has an inner diameter of $300 \mathrm{~mm}$ and a thickness of $10 \mathrm{~mm}$. The outer diameter of the guiding tube becomes the inner diameter of coil, which is $320 \mathrm{~mm}$. The schematic diagram of the coil and projectile 


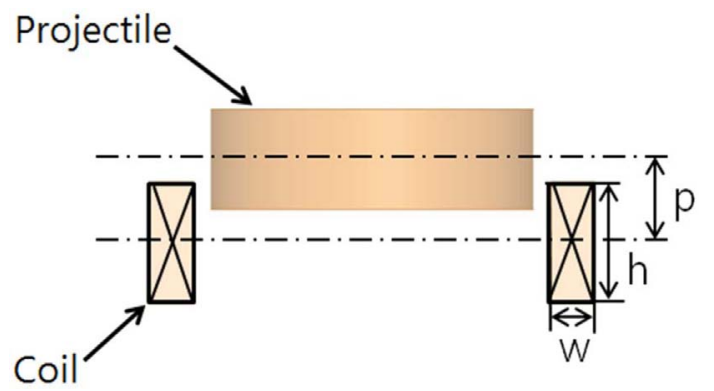

Fig. 1. (Color online) 2D Schematic diagram of coil and projectile.

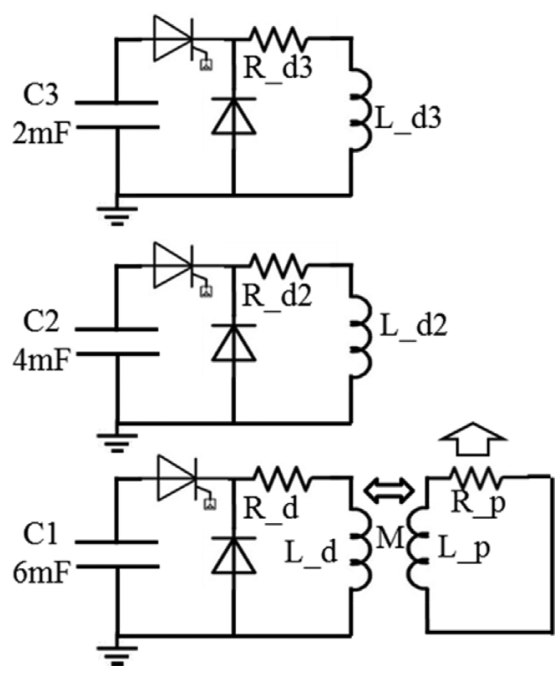

Fig. 2. Circuit model of 3-stage EIL.

is shown in Fig. 1 in which $w, h$, and $p$ indicate the coil width, coil height, and the distance between coil center and projectile center, respectively.

The simplified circuits of the 3-stage EIL system are shown in Fig. 2. A $600 \mathrm{~kJ}$ pulsed power system (PPS) for electromagnetic launcher experiment is considered as a power source for driving coils [7, 8]. The capacitance of the PPS will be modified to $6 \mathrm{mF}, 4 \mathrm{mF}$, and $2 \mathrm{mF}$ for the first, the second, and the third stage, respectively, considering that faster projectiles needs shorter pulse widths. The $6 \mathrm{kV}$ charging voltage is switched with thyristor switches and crowbarred with diodes. The resistance of the coil, R_coil, has been calculated with copper winding wire geometries and the inductance of the coil, L_coil, has been calculated through the simulation.

\subsection{Axial force analysis}

The total energy stored in the EIL can be described as follows [9];

$$
W_{m}=\frac{1}{2} L_{p} i_{p}^{2}+\frac{1}{2} L_{p} i_{p}^{2}+M i_{p} i_{d}
$$

where subscripts $p$ and $d$ denote the projectile and driving coil, respectively, and $M$ is the mutual inductance between the coil and the projectile. $M$ is not a constant in time because axial motion of the projectile affects the induction current on its surface. For the simplicity of the calculation, however, if we supposed that the changes of currents, $i_{d}$ and $i_{p}$, due to the motion are negligible, the force could be described as follows;

$$
F_{p}=\frac{d W_{m}}{d z}=\frac{d M}{d z} i_{p} i_{d}
$$

According to Kirchhoff's voltage law, the circuit equation of the first stage in Fig. 2 can be obtained as

$$
\begin{aligned}
& \frac{1}{C} \int i_{d} d t+R_{d} i_{d}+L_{d} \frac{d i_{d}}{d t}+M \frac{d i_{p}}{d t}=0, \\
& R_{p} i_{p}+M \frac{d i_{d}}{d t}+L_{p} \frac{d i_{p}}{d t}=0,
\end{aligned}
$$

where $R_{d}$ and $R_{p}$ are resistances of the driving coil and the projectile, respectively. The induced current $\left(i_{p}\right)$ on the projectile is obtained by solving equations (3) and (4).

$$
i_{p}(t)=\frac{M}{L_{p}}\left(\frac{R_{p}}{L_{p}} e^{-\frac{R_{p}}{L_{p}} t}-1\right) i_{d}(t) .
$$

By using the relations between mutual inductance and self-inductance,

$$
M=k \sqrt{L_{d} L_{p}}, k \text { : coupling coefficient. }
$$

$i_{p}$ can be expressed as equation (7).

$$
i_{p}(t)=k \sqrt{\frac{L_{d}}{L_{p}}}\left(\frac{R_{p}}{L_{p}} e^{-\frac{R_{p}}{L_{p}} t}-1\right) i_{d}(t) .
$$

We can see the projectile current is proportional to the coupling coefficient and the square root of inductance ratio of $L_{d} / L_{p}$. Substitution of equation (7) into equation (2) gives the relationship between axial propulsion force $F_{p}$ and driving coil current $i_{d}$.

$$
F_{p}(t)=\frac{d M}{d z} \frac{M}{L_{p}}\left(\frac{R_{p}}{L_{p}} e^{-\frac{R_{p}}{L_{p}} t}-1\right) i_{d}^{2}(t) .
$$

Quantitative analysis of equation (8) in a real EIL system is very difficult. Though mutual inductance was assumed to be a constant value in driving equation (8), it changes with time as the projectile moves along the acceleration tube, in the real system. Furthermore, in a multi-stage acceleration system, the analytic solution is nearly impossible to get since the neighboring coils interact with the projectile in a complicated way. For this reason, 


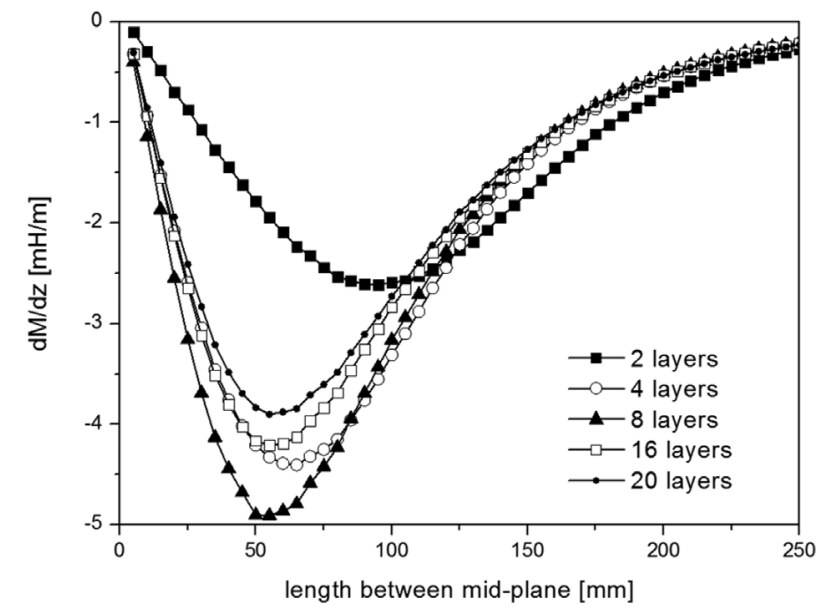

Fig. 3. Calculation result of $\mathrm{dM} / \mathrm{dz}$ for 80 -turn coil with different layers.

analysis of multi-stage EIL is conducted by using a special code or commercial software based on the FEA method in many studies [11-13]. In this study, we designed a threestage EIL system by using electromagnetic analysis software (Maxwell 2D, Ansys Electromagnetics Suite 16.2) based on the FEA method.

\subsection{Calculation of $\mathrm{dM} / \mathrm{dz}$}

As shown in equation (7), the value of $d M / d z$ is an important factor that determines the driving force of the EIL system. The efficiency of energy transfer depends on the relation between the coil current and the curve of $\mathrm{dM} /$ $\mathrm{dz}$.

Figure 3 shows the calculation results of $\mathrm{dM} / \mathrm{dz}$ for an 80-turn coil with different layers, as an example. At the same turns of the coil, as the layer increases, the height, $h$, becomes shorter, and the width, w, becomes thicker. The values of $\mathrm{dM} / \mathrm{dz}$ are function of the relative central distance between the coil and the projectile along the $\mathrm{z}$-axis and have maximum absolute value at a certain distance.

On the other hand, the calculation shows that the maximum absolute value of $\mathrm{dM} / \mathrm{dz}$ depends on the layers. In case of 80-turns, the maximum absolute value of $\mathrm{dM} /$ $\mathrm{dz}$ was $5 \mathrm{mH} / \mathrm{m}$ with 8 layers, which is regarded as the optimum layers for that number of turns. For every 40 turns from 40 to 280 , the optimum layers have been estimated to be $4,8,8,10,10,15$, and 14 , respectively.

\subsection{Optimum design of driving coils}

We have designed the coils for the 3-stage EIL system by optimizing each stage coil independently of other stage coils in order to save calculation time. Therefore, the initial velocity of the projectile at the second stage is the final velocity of the first stage, and the initial third stage velocity, is the final velocity of the second stage.

The wire for the coils has a cross section of $10 \mathrm{~mm}^{2}(5$ $\mathrm{mm} \times 2 \mathrm{~mm}$ ) and an insulation thickness of $0.25 \mathrm{~mm}$. The calculation parameters selected for coil optimization are the number of turn, layers, and the initial position of the projectile against the coils. The numbers of turn were calculated from 40 to 280 with interval of 40 turns. The layers were calculated up to optimum condition of $\mathrm{dM} / \mathrm{dz}$, which was described in the previous section. The initial positions of the projectile, $\mathrm{p}$ were calculated from -45 $\mathrm{mm}$ to $45 \mathrm{~mm}$ along the z-axis with intervals of $10 \mathrm{~mm}$ considering the trends of the final velocities.

The electromagnetic simulation program was run with these conditions. From the calculation results, the optimized first stage coil was estimated to be 120 turns, 5 layers, and $35 \mathrm{~mm}$ of position with resistance and inductance of $215.6 \mathrm{~m} \Omega$ and $5.31 \mathrm{mH}$, respectively. The velocity at $1 \mathrm{~m}$, which is considered as the final velocity of the optimized coil, was calculated to be $27.3 \mathrm{~m} / \mathrm{s}$. This becomes the initial velocity of the projectile for the second stage calculation. By the same process with the first stage, the optimized second coil was estimated to be 80 turns, 4 layers, and $-15 \mathrm{~mm}$ of position which means that the center of the projectile is located $15 \mathrm{~mm}$ below the center of the coil. The final velocity of the second coil was calculated to be $37.8 \mathrm{~m} / \mathrm{s}$. The resistance and inductance were estimated to be $142.65 \mathrm{~m} \Omega$ and $2.56 \mathrm{mH}$, respectively.

The third stage coil was determined by the same procedure and estimated to be 80 -turns, 4 layers, and $-15 \mathrm{~mm}$ position with $142.65 \mathrm{~m} \Omega$ and $2.56 \mathrm{mH}$. The final velocity of the third stage was calculated to be $42.57 \mathrm{~m} / \mathrm{s}$. The optimized coil design parameters and their calculation results are summarized in Table 1. Figure 4 shows the final velocities as a function of triggering position with the optimized turns and layers. Figure 5 shows the

Table 1. Summary of simulation results.

\begin{tabular}{lccc}
\hline \hline Voltage $=6 \mathrm{kV})$ & $1^{\text {st }}$ stage & $2^{\text {nd }}$ stage & $3^{\text {rd }}$ stage \\
\hline Turns & 120 & 80 & 80 \\
Layers & 5 & 4 & 4 \\
$\mathrm{w}$ (thickness of coil) $[\mathrm{mm}]$ & 132 & 110 & 110 \\
$\mathrm{~h}$ (height of coil) [mm] & 12.5 & 10 & 10 \\
$\mathrm{p}$ (triggering position) $[\mathrm{mm}]$ & 35 & -15 & -15 \\
Resistance $[\mathrm{m} \Omega]$ & 215.6 & 142.65 & 142.65 \\
Inductance $[\mathrm{mH}]$ & 5.31 & 2.56 & 2.56 \\
Capacitance $[\mathrm{mF}]$ & 6 & 4 & 2 \\
Initial Velocity $[\mathrm{m} / \mathrm{s}]$ & 0 & 27.29 & 37.80 \\
Velocity at $1 \mathrm{~m}[\mathrm{~m} / \mathrm{s}]$ & 27.29 & 37.80 & 42.57 \\
peak current $[\mathrm{kA}]$ & 5.66 & 6.40 & 4.77 \\
peak force for $\mathrm{z}[\mathrm{kN}]$ & 283.96 & 596.59 & 120.98 \\
\hline
\end{tabular}




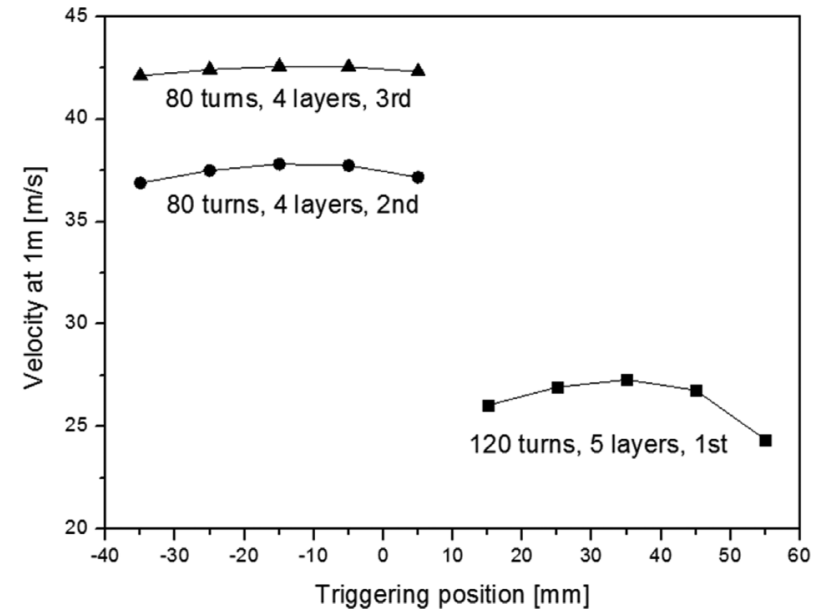

Fig. 4. Projectile velocities at each stage as a function of triggering position.

current, force and velocity of the each stage coil that was optimized independently of the other stage coils.

\subsection{Modelling of 3-stage EIL}

For the optimized coil design, we have assumed that the coils are independent of each other. However, the interval between the coils should be limited in real systems, and the electromagnetic interaction between coils cannot be ignored. For the investigation of interaction of the coils we have set three intervals $(200 \mathrm{~mm}, 50 \mathrm{~mm}$, and $0 \mathrm{~mm}$ as shown in Fig. 6).

It could be considered that the $200 \mathrm{~mm}$ interval is enough to be free from interaction with other coils because the value of $\mathrm{dM} / \mathrm{dz}$ at $200 \mathrm{~mm}$ interval is around $10 \%$ of its maximum, as shown in Fig. 3. The second and third coils were fired at $14.8 \mathrm{~ms}$ and $23.8 \mathrm{~ms}$, respectively. These triggering times are the times that the projectile reaches the position we considered optimum from the calculation results of the previous section. The time variations of current, force, and velocity are shown in Fig. 7 (a). It can be seen that the velocity is slightly decreasing at the triggering times of second and third coils.

In case of $50 \mathrm{~mm}$ of interval, the triggering times of the second and third coils were $9.3 \mathrm{~ms}$ and $14.3 \mathrm{~ms}$, respectively. Figure $7(\mathrm{~b})$ shows the calculation result for this case. Compared with the $200 \mathrm{~mm}$ interval case, the velocity is slightly increasing at the triggering times of the second and third coils. The final velocity also has been increased.

When there is no interval between the coils, the triggering times of the second and third coils were $7.5 \mathrm{~ms}$ and $11.2 \mathrm{~ms}$, respectively and the results of calculation are shown in Fig. 7(c). The tendency of velocity increase is
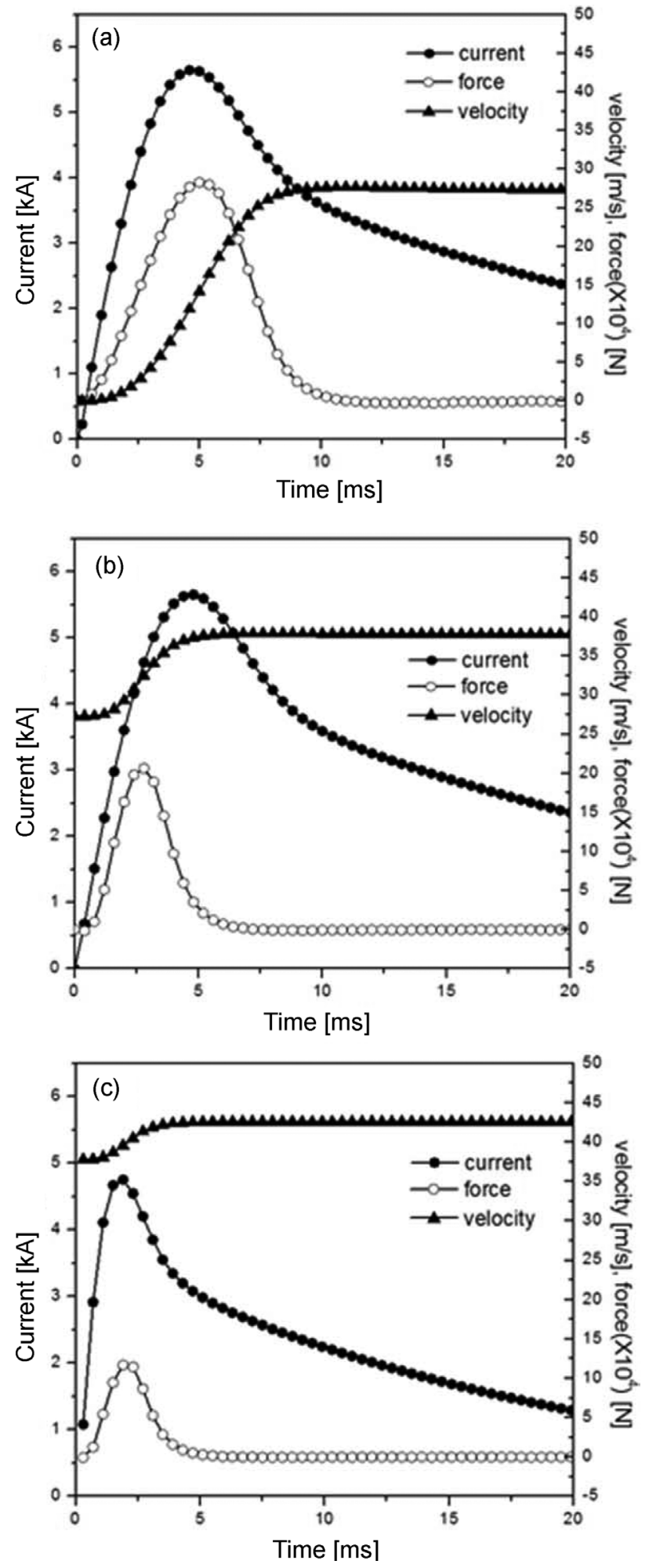

Fig. 5. Current, force, and velocity of optimized coil (a) $1^{\text {st }}$ stage, (b) $2^{\text {nd }}$ stage, and (c) $3^{\text {rd }}$ stage. Each stage coil was optimized independently of the other stage coils.

nearly constant at the triggering times of the second and third coils, which results in the highest final velocity 


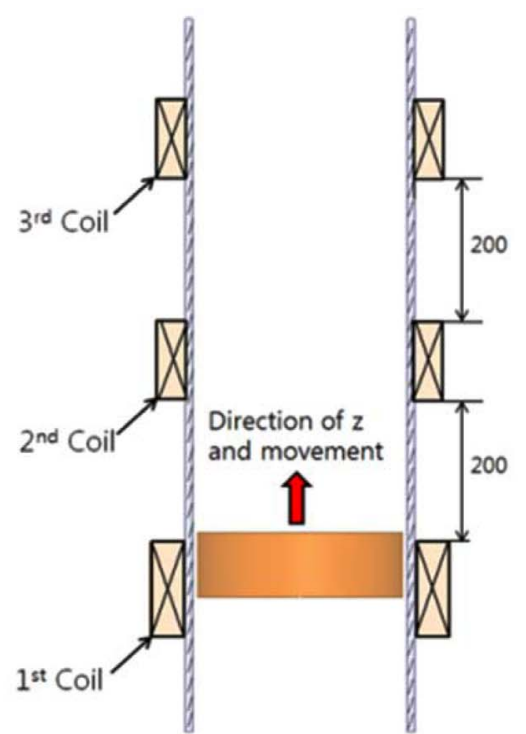

(a)

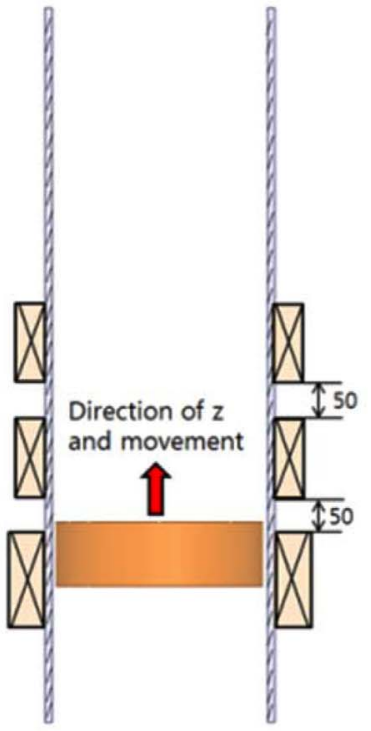

(b)

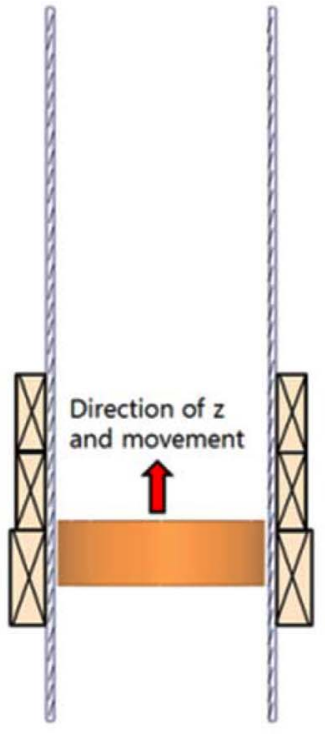

(c)
Fig. 6. (Color online) Schematic views of 3-stage EIL. The distances between coils are (a) $200 \mathrm{~mm}$, (b) $50 \mathrm{~mm}$, and (c) $0 \mathrm{~mm}$.

among the three intervals.

\section{Discussion}

As mentioned, when we design individual coils we optimized them with a given initial velocity. When three coils are arranged with $200 \mathrm{~mm}, 50 \mathrm{~mm}$, and $0 \mathrm{~mm}$ distance between them, the velocities of the projectile at the exit of each coils show different values compared to individual coil design. Table 2 summarizes the projectile velocity comparison between coil arrangements. The efficiency indicates the ratio of kinetic energy of the projectile to the
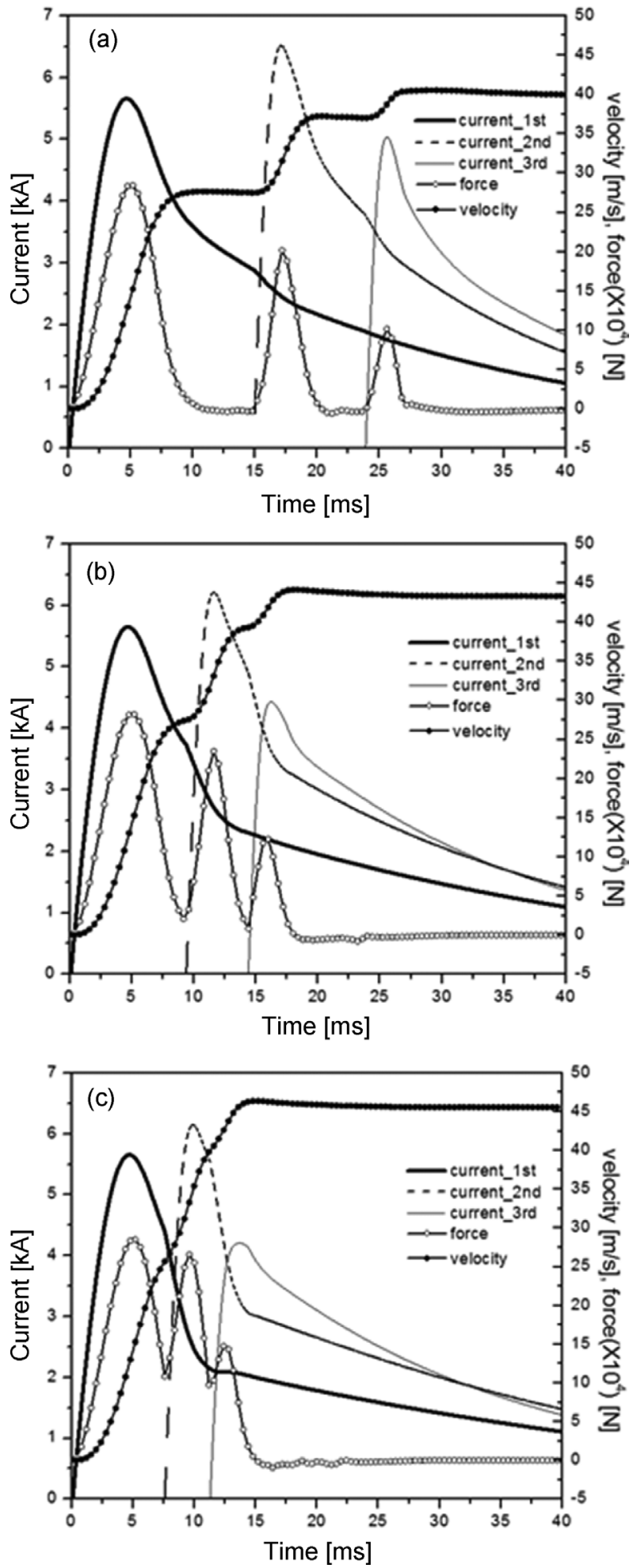

Fig. 7. Current, force, and velocity of 3 -satge EIL. The distances between coils are (a) $200 \mathrm{~mm}$ (b) $50 \mathrm{~mm}$, and (c) $0 \mathrm{~mm}$.

discharge energy of a driving power module.

Small differences in velocities after acceleration of the 1st coil arises from a difference in reference time of 
Table 2. Calculation results of the velocities and efficiencies for each case.

\begin{tabular}{llcccc}
\hline \hline & & $1^{\text {st }}$ coil & $2^{\text {nd }}$ coil & $3^{\text {rd }}$ coil & total \\
\hline Individual & Velocity [m/s] & 27.29 & 37.8 & 42.57 & 42.57 \\
design & efficiency [\%] & 17.24 & 23.75 & 26.64 & 20.97 \\
\hline (a) $200 \mathrm{~mm}$ & velocity [m/s] & 27.5 & 37.01 & 39.99 & 39.99 \\
separation & efficiency [\%] & 17.51 & 21.29 & 15.94 & 18.51 \\
\hline (b) $50 \mathrm{~mm}$ & velocity [m/s] & 27.43 & 39.3 & 43.32 & 43.32 \\
separation & efficiency [\%] & 17.42 & 27.5 & 23.08 & 21.72 \\
\hline (c) $0 \mathrm{~mm}$ & velocity [m/s] & 25.47 & 39.82 & 45.58 & 45.58 \\
separation & efficiency [\%] & - & - & - & 24.05 \\
\hline
\end{tabular}

measurement. The reference time is the switch-on time of the 2nd and 3rd coil for each arrangement. In case of (a), the distance between coils was $200 \mathrm{~mm}$ in order to ensure independent operation of the driving coils without interference from the other coils. The final velocity of case (a) is smaller than that of individual coil design. In the case of (b) and (c), the final velocity is larger than those of individual design case or case (a), although the distances between the coils are much smaller than case (a). In order to understand the velocity dependency on the distance between coils, we investigated the variation of induced current in the projectile. Figure 8 shows calculation results of projectile current for 1st and 2nd stage acceleration with coil distance of $200 \mathrm{~mm}$ and $50 \mathrm{~mm}$. The second coil of $50 \mathrm{~mm}$ distance was triggered earlier than that of $200 \mathrm{~mm}$ for the best efficiency. We can see

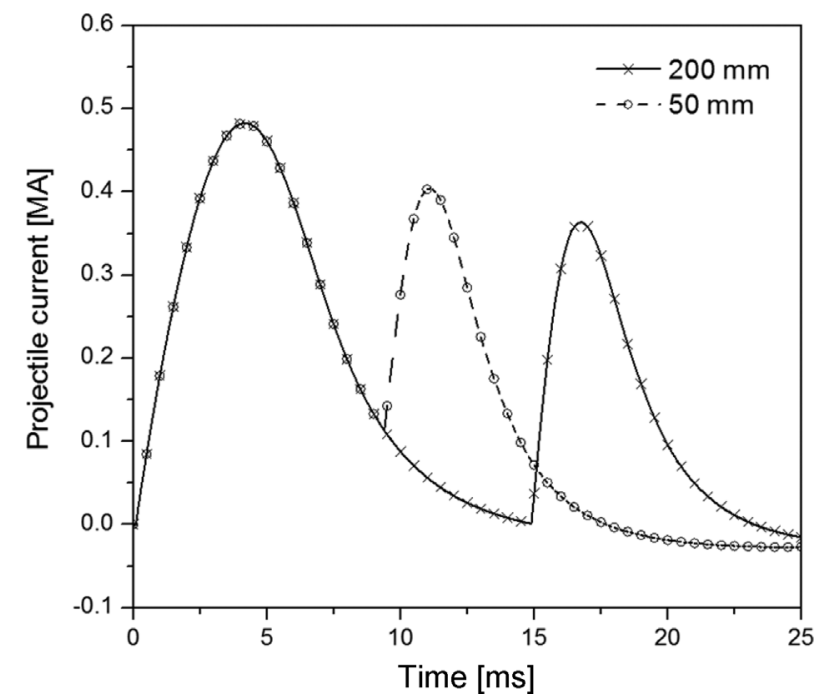

Fig. 8. The current profiles induced in the projectile. The distances between the $1^{\text {st }}$ and the $2^{\text {nd }}$ coils are $200 \mathrm{~mm}$ and 50 $\mathrm{mm}$. that the second peak of the projectile current of $50 \mathrm{~mm}$ is $10 \%(0.40 \mathrm{MA} / 0.36 \mathrm{MA})$ higher than that of $200 \mathrm{~mm}$. As the distance between coils become shorter, the projectile current is increased at the time of the second coil trigger, and thereby a higher drive force can be obtained.

This superposition effect of the projectile current by the adjacent coil current is considered to be the reason for higher acceleration efficiency with a shorter coil distance.

\section{Conclusions}

A 3-stage EIL to demonstrate the launch of a $290 \mathrm{~mm}$ diameter, $50 \mathrm{~kg}$ mass projectile has been designed. For the optimal design of the EIL, various parameters were analyzed. The final projectile velocity and energy conversion efficiency was $45.58 \mathrm{~m} / \mathrm{s}$ and $24 \%$, respectively, when the total stored energy of capacitors was $216 \mathrm{~kJ}$. The fabrication of a 3-stage EIL is undergoing and a comparison study between experimental results and simulation results will be conducted in near future.

\section{References}

[1] K. McKinney and P. Mongeau, IEEE Trans. Magn. 20, 239 (1984).

[2] R. Kaye, B. Turman, M. Aubuchon, D. Lamppa, G. Mann, Edward van Reuth, K. Fulton, G. Malejko, P. Magnotti, D. Nguyen, D. Borgwarth, A. Johnson, and R. Poppe, in Proc. 16th IEEE Int. Pulsed Power Conf., 1810 (2007).

[3] M. S. Aubuchont, T. R. Lockner, and B. N. Turman, in Proc. 15th IEEE Int. Pulsed Power Conf., 75 (2005).

[4] B. D. Skurdal and R. L. Gaigler, IEEE Trans. Magn. 45, 458 (2009).

[5] S. Barmada, A. Musolino, M. Raugi, and R. Rizzo, IEEE Trans. Magn. 37, 111 (2001).

[6] R. J. Kaye, IEEE Trans. Magn. 41, 194 (2005).

[7] Y. S. Jin, Y. B. Kim, J. S. Kim, C. Cho, S. W. Lim, B. Lee, S. H. Kim, S. An, S. H. Yoon, and I. S. Koo, IEEE Trans. Plasma Sci. 41, 2671 (2013).

[8] B. Lee, S. An, S. H. Kim, Y. H. Lee, K. S. Yang, Y. S. Jin, Y. B. Kim, J. S. Kim, C. Cho, S. H. Yoon, and I. S. Koo, IEEE Trans. Plasma Sci. 42, 2886 (2014).

[9] H. Kolm and P. Mongeau, IEEE Trans. Magn. 20, 227 (1984).

[10] S. Babic, F. Sirois, C. Akyel, G. Lemarquand, V. Lemarquand, and R Ravaud, IEEE Trans. Magn. 47, 2034 (2011).

[11] K. A. Polzin, J. E. Adwar, and A. K. Hallock, IEEE Trans, Magn. 49, 1453 (2013).

[12] S. Madhavan, C. D. Sijoy, S. Pahari, S. Chaturvedi, and IPF Team, IEEE Trans. Plasma Sci. 42, 323 (2014).

[13] X. Tao, S. Wang, Y. Huangfu, S. Wang, and Y. Wang, IEEE Trans. Plasma Sci. 43, 1208 (2015). 